\title{
The Central Role of Chromatin in Autoimmune Responses to Histones and DNA in Systemic Lupus Erythematosus
}

\author{
Rufus W. Burlingame, Mee L. Boey, ${ }^{\star}$ Gordon Starkebaum, ${ }^{\star}$ and Robert L. Rubin \\ W. M. Keck Autoimmune Disease Center, Department of Molecular and Experimental Medicine, The Scripps Research Institute, La Jolla, \\ California 92037; *Department of Rheumatology and Immunology, Tan Tock Seng Hospital, Singapore; and ${ }^{\ddagger}$ Seattle Veterans Affairs \\ Medical Center and Department of Medicine, University of Washington, Seattle, Washington 98108
}

\begin{abstract}
To gain insight into the mechanisms of autoantibody induction, sera from 40 patients with systemic lupus erythematosus (SLE) were tested by ELISAs for antibody binding to denatured individual histones, native histone-histone complexes, histone-DNA subnucleosome complexes, three forms of chromatin, and DNA. Whole chromatin was the most reactive substrate, with $88 \%$ of the patients positive. By chi-square analysis, only the presence of anti-(H2AH2B ), anti-[(H2A-H2B) -DNA], and antichromatin were correlated with kidney disease measured by proteinuria $>0.5 \mathrm{~g} / \mathrm{d}$. SLE patients could be divided into two groups based on their antibody-binding pattern to the above substrates. Antibodies from about half of the patients reacted with chromatin and the (H2A-H2B) -DNA subnucleosome complex but displayed very low or no reactivity with native DNA or the (H3-H4) ${ }_{2}$-DNA subnucleosome complex. An additional third of the patients had antibody reactivity to chromatin, as well as to both subnucleosome structures and DNA. Strikingly, all sera that bound to any of the components of chromatin also bound to whole chromatin, and adsorption with chromatin removed $85-100 \%$ of reactivity to (H2A-H2B) -DNA, (H3-H4) $)_{2}-$ DNA, and native DNA. Individual sera often bound to several different epitopes on chromatin, with some epitopes requiring quaternary protein-DNA interactions. These results are consistent with chromatin being a potent immunogenic stimulus in SLE. Taken together with previous studies, we suggest that antibody activity to the (H2A-H2B)-DNA component signals the initial breakdown of immune tolerance whereas responses to $(\mathrm{H3}-\mathrm{H} 4)_{2}-$ DNA and native DNA reflect subsequent global loss of tolerance to chromatin. (J. Clin. Invest. 1994. 94:184-192.) Key words: lupus erythematosus, systemic • autoantibodies • histones, immunology • enzymelinked-immunosorbent assay
\end{abstract}

\section{Introduction}

Patients with systemic lupus erythematosus (SLE) have a diverse array of antinuclear autoantibodies $(1,2)$. The most com-

Address correspondence to Robert L. Rubin, Ph.D., Department of Molecular and Experimental Medicine-SBR6, The Scripps Research Institute, 10666 N. Torrey Pines Road, La Jolla, CA 92037.

Received for publication 17 August 1993 and in revised form 18 February 1994.

J. Clin. Invest.

(C) The American Society for Clinical Investigation, Inc. 0021-9738/94/07/0184/09 \$2.00

Volume 94, July 1994, 184-192 mon of these react with histones (3-7), native DNA (nDNA), ${ }^{1}$ denatured DNA (dDNA) (8-10), Sm antigen, and ribonucleoprotein particles $(11,12)$.

The origin of these autoantibodies is enigmatic. Previous studies hypothesized that autoimmunization with the self antigen is the induction mechanism for certain autoantibodies because it can account for their reactivity with multiple epitopes on native forms of the antigen $(2,12)$ and their stronger binding to self antigen than to antigens from animals further removed on the evolutionary tree (13). Productive somatic mutations in the genes encoding their hypervariable regions of $\mathrm{Ig}$ also indicate selection by antigen $(14,15)$. However, conventional immunization with autoantigens generally fails to yield an immune response to the native immunogen (16). This is particularly true for histones (17) and nDNA (18). Thus other hypotheses, such as molecular mimicry (19), cross-reaction with a bacterial antigen (20), polyclonal activation (21), and breakdown of an idiotypic network (22) have also been proposed.

To gain insight into the genesis of autoantibodies, we analyzed the epitopes on chromatin recognized by antibodies from patients with SLE. This is an attractive model system because indirect evidence from experiments with the LE cell assay (23, 24 ), soluble deoxyribonucleoprotein (25), and histone-DNA reconstitution $(3,26)$ suggested that antibodies to chromatin, as well as to histones and DNA, are common in patients with SLE. Additionally, the antigenic regions of chromatin can be finely mapped because the structure $(27,28)$ and biochemical properties $(29,30)$ of chromatin and its components are well known and can be readily isolated. Chromatin would be a candidate for a principal autoimmunogen in SLE if it has the epitope diversity to bind autoantibodies to histones and nDNA, as well as antibodies reacting with structures produced by histoneDNA quaternary interactions.

For this study, all known forms of histones and histoneDNA complexes were compared for antigenicity using previously described ELISAs (31). The antigens included $(a)$ denatured individual histones $\mathrm{H} 2 \mathrm{~A}, \mathrm{H} 2 \mathrm{~B}, \mathrm{H} 3, \mathrm{H} 4$, and $\mathrm{H} 1$; $(b)$ native histone-histone complexes of the $\mathrm{H} 2 \mathrm{~A}-\mathrm{H} 2 \mathrm{~B}$ dimer and $(\mathrm{H} 3-\mathrm{H} 4)_{2}$ tetramer; and $(c)$ subnucleosome complexes of $\mathrm{H1}$ DNA, (H2A-H2B) -DNA and (H3-H4) $)_{2}$-DNA. Three forms of chromatin also were used: whole (unextracted) chromatin; $\mathrm{H} 1$-stripped chromatin in which $\mathrm{H} 1$ and most nonhistone proteins were removed by extraction with $0.5 \mathrm{M} \mathrm{NaCl}$ (32); and trypsinized $\mathrm{H} 1$-stripped chromatin in which $\sim 15 \%$ of the $\mathrm{NH}_{2-}$ terminal regions of all core histones and some COOH-terminal amino acids of $\mathrm{H} 2 \mathrm{~A}$ and $\mathrm{H} 3$ were removed by enzymatic digestion (33). We found that sera from SLE patients recognized

1. Abbreviations used in this paper: dDNA, denatured DNA; dimerDNA, (H2A-H2B) -DNA complex; nDNA, native DNA; tetramerDNA, $(\mathrm{H} 3-\mathrm{H} 4)_{2}$ tetramer-DNA complex. 
many epitopes on chromatin, some of which required quaternary structures for expression of antigenicity. Anti-DNA antibodies were a subset of this wide spectrum of antichromatin autoantibodies. Along with previous observations on the kinetics of appearance of antichromatin antibodies of similar specificity in murine lupus, the present results suggest that autoimmunization with chromatin accompanied by sequential loss of tolerance to certain of its numerous epitopes underlies this autoimmune response.

\section{Methods}

Patients. The sera of 40 patients from the lupus clinic of Tan Tock Seng Hospital, Singapore, who met the revised criteria of the American College of Rheumatology for SLE (34) were studied. All had active lupus when their blood was drawn. 24 patients had central nervous system (CNS) involvement and had been treated for various lengths of time. The other 16 patients were only recently diagnosed and were untreated. The occurrences of the 10 recorded symptoms were as follows: 17 of 40 with malar rash, 1 of 40 with discoid rash, 16 of 40 with alopecia, 13 of 40 with photosensitivity, 8 of 40 with oral ulcers, 25 of 40 with arthritis, 21 of 40 with proteinuria $>0.5 \mathrm{~g} / \mathrm{d}, 28$ of 40 with neurological abnormalities, 23 of 40 with hematologic abnormalities, and 9 of 40 with serositis. A detailed analysis of nonchromatin-binding autoantibodies from some of the patients with CNS involvement has been reported previously (35). The ethnic makeup of this group was 31 Chinese, 6 Malaysian, 2 Indian, and 1 Euroasian.

Another group of 37 patients with established SLE was subjected to a more limited study. Sera were collected in the offices of private rheumatologists in Seattle, Washington and from the arthritis clinics of the University of Washington Affiliated Hospitals and the Seattle Veterans Affairs Medical Center. Many of these patients were on a variety of medications including corticosteroids in various doses. All patients fulfilled at least four criteria for SLE (34). Half of the patients (18/37) had hematologic manifestations, but only two patients had neurological disease. The ethnic makeup of this group was 21 white, 5 black, 2 Hispanic, 1 Japanese, 1 Laotian, and 7 unrecorded.

Antigens. Procedures for isolating chromatin and histones and reconstituting histone-DNA subnucleosome complexes have been described in detail (31). Briefly, nuclei were isolated from calf thymus (Pel Freeze Biologicals, Rogers, AR), and $\mathrm{Hl}$ and histone-histone complexes were prepared by salt extraction of nuclei at neutral $\mathrm{pH}$ followed by column chromatography on CM-52 cellulose (Whatman, Maidstone, England) (29). Histone-DNA subnucleosome complexes were reconstituted by high to low salt dialysis (36). Individual histones were prepared from the appropriate histone-histone complex using P60 (Bio-Rad Laboratories, Inc., Richmond, CA) column chromatography (37). Whole chromatin, H1-stripped chromatin, and trypsinized chromatin were prepared as described $(32,33)$, and the quality of the preparations was the same as reported previously $(31,38)$. Native calf thymus DNA (Calbiochem Corp., La Jolla, CA) was further purified by digestion with proteinase $\mathrm{K}$, extraction with phenol, and digestion with S1 nuclease. dDNA was prepared by placing nDNA in a boiling water bath for $15 \mathrm{~min}$ and cooling on ice. Tetanus toxoid USP (Connaught Laboratories Inc., Swiftwater, PA) was diluted 1:50 in PBS before use in ELISA.

ELISA. The ELISAs were performed as described (31) with the modification that polystyrene plates were precoated with methylated BSA for the dDNA as well as nDNA assays. Briefly, most substrates (see reference 38 for exceptions) were dissolved in PBS at $2.5 \mu \mathrm{g} / \mathrm{ml}$, and $200 \mu \mathrm{l}$ of each solution was added to an microtiter plate (Immulon 2; Dynatech Laboratories, Inc., Chantilly, VA), incubated overnight, and blocked for $2 \mathrm{~h}$ with $0.1 \%$ gelatin in PBS. Samples diluted 1:400 in serum diluent $(0.1 \%$ gelatin, $0.1 \%$ BSA, $0.075 \%$ bovine gamma globulin, $0.05 \%$ Tween-20 in PBS) were reacted in duplicate for $2 \mathrm{~h}$, washed with PBS- $0.05 \%$ Tween, and detected with horseradish peroxidase-conjugated goat anti-human IgM or IgG (Caltag Laboratories, South San Francisco, CA). The anti-IgM and anti-IgG conjugates were diluted so that they produced similar OD on ELISA plates coated with purified IgM or IgG, respectively. The OD of the samples was read on a spectrophotometer (MR600; Dynatech Laboratories, Inc.) at $60 \mathrm{~min}$ after addition of the colorimetric reagent and, if this reading was greater than the sensitivity of the spectrophotometer (2.0 OD), the final OD was calculated by extrapolation from an earlier reading. Extrapolation was linear to at least $13 \mathrm{OD}$, thereby expanding the dynamic range of the assay as previously described (31). The reactivity with each antigen of 15 laboratory and clerical personnel was determined, and this average OD plus 2 SD was subtracted from the reactivity of each patient's serum on that antigen, yielding "OD above normal." The correction for normal serum binding on different antigens varied from $0.03-0.15$ OD for IgG and $0.04-0.43$ OD for IgM.

Solid phase antibody adsorption. Diluted sera were incubated four times for $1 \mathrm{~h}$ each in multiple wells of an ELISA plate coated with either nDNA or whole chromatin. Controls consisted of the sera similarly adsorbed in gelatin-coated wells.

Blocking of murine antibodies. A human serum at 1:300 dilution was preincubated for $2 \mathrm{~h}$ with chromatin bound to the ELISA plate. Subsequently, the same human serum at 1:30 dilution was coincubated with a serum from an MRL/lpr or BXSB autoimmune mouse (39), or a control monoclonal antihistone (40) or anti-nDNA (41) antibody. Mouse antibody was detected with horseradish peroxidase-conjugated goat anti-mouse Ig that had been preadsorbed with human Ig (Caltag Laboratories).

Statistics. For each patient, the correlation coefficient was calculated relating the amount of each autoantibody to all other autoantibodies, and the results averaged among all patients for each comparison. The correlation between the presence of an IgG antibody and a symptom was determined by chi-square analysis with Yates correction. Other analyses were performed by two-tailed $t$ test.

\section{Results}

Comparison of individuals with and without $\operatorname{IgG}$ anti-nDNA autoantibodies. The profiles of IgG binding of the 40 sera in the predominantly Chinese SLE patients on the 15 substrates suggested four groups: 14 patients showed strong ( $>0.5$ OD above normal) binding to chromatin and nDNA; 14 displayed strong binding to chromatin but low or none to nDNA; 7 patients showed low binding to chromatin and a few other substrates; the remaining 5 sera were not above normal on any substrate. There were no significant differences by two-tailed $t$ test in antibody binding to any of the substrates between the patients treated with prednisone and the newly diagnosed, untreated patients.

The IgG binding pattern for the 11 sera reacting most strongly with nDNA is shown in Fig. $1 A$. Reactivity to whole chromatin, H1-stripped chromatin, and (H2A-H2B)-DNA predominated, but all other DNA-containing substrates also were bound strongly. The majority of these sera bound to the individual histones $\mathrm{H} 1, \mathrm{H} 2 \mathrm{~A}$, and $\mathrm{H} 2 \mathrm{~B}$ and relatively less with $\mathrm{H} 3$ and $\mathrm{H} 4$. Four of these 11 sera showed a large increase in binding to the $\mathrm{H} 2 \mathrm{~A}-\mathrm{H} 2 \mathrm{~B}$ dimer complex compared with $\mathrm{H} 2 \mathrm{~A}$ or $\mathrm{H} 2 \mathrm{~B}$, whereas only one serum demonstrated a small increase for $(\mathrm{H} 3-\mathrm{H} 4)_{2}$ compared to $\mathrm{H} 3$ and $\mathrm{H} 4$. All but one serum bound more strongly to nDNA than to dDNA. The hierarchy of binding to these substrates was similar for all individuals whether they had a high or low titer of antichromatin autoantibodies, yielding a pattern in which very few of the lines crossed (Fig. $1 \mathrm{~A}$ ).

The binding pattern of the 11 sera with the highest IgG reactivity to chromatin but no or very low reactivity to nDNA is displayed in Fig. $1 B$. The overall antichromatin and antihistone 

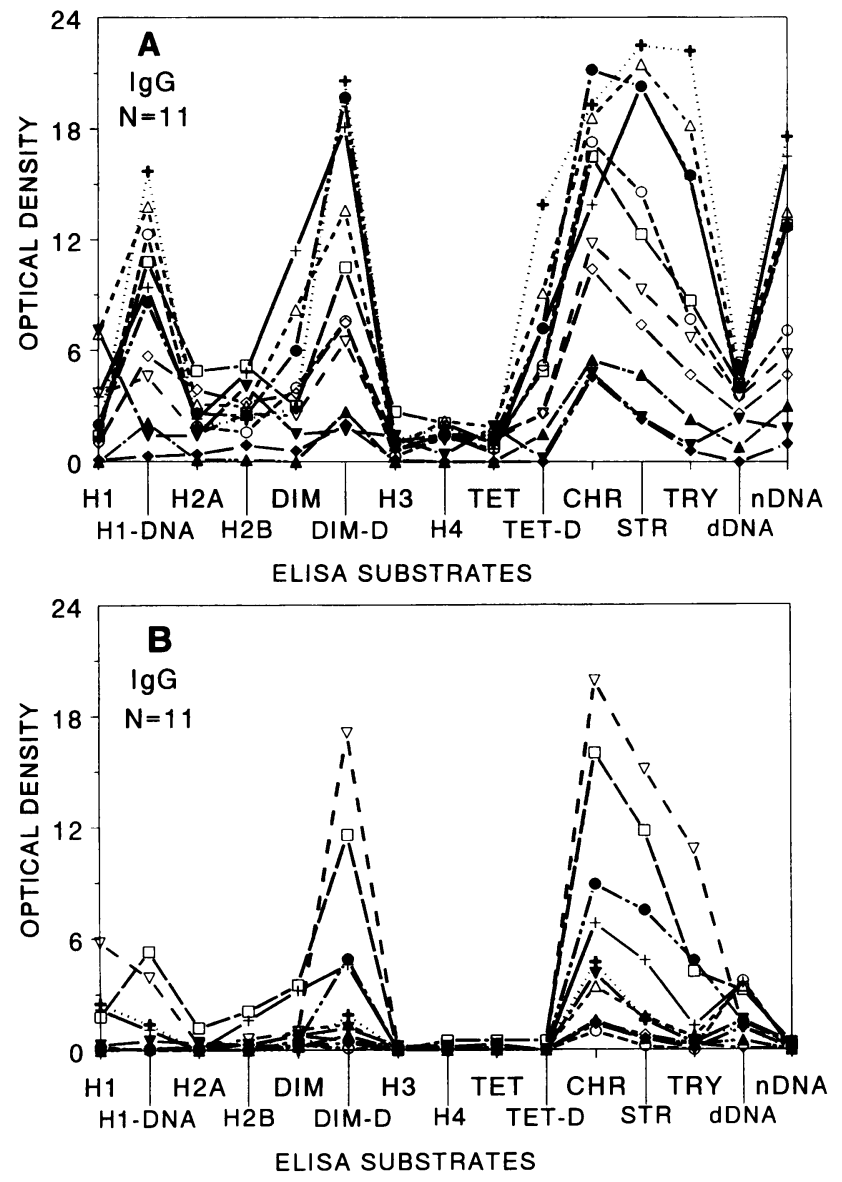

Figure 1. IgG autoantibodies to components of chromatin in SLE. The data points connected by a line are the activities (OD) of a serum on the indicated substrates after correcting for normal serum binding. $(A)$ IgG activity in patients with IgG anti-nDNA antibodies. $(B)$ IgG activity in patients without IgG anti-nDNA antibodies. The two figures are drawn to the same scale. The abbreviations for the substrates are DIM, H2AH2B dimer; DIM-D, (H2A-H2B) -DNA subnucleosome complex; $T E T$, (H3-H4) $)_{2}$ tetramer; TET-D, (H3-H4) $)_{2}$ DNA complex; $C H R$, whole, unextracted chromatin; STR, H1-stripped chromatin; TRY, trypsinized H1-stripped chromatin. Previous studies demonstrated the validity of comparing antibody activities on different immobilized antigens based on analysis of the amount of antigen on the solid phase (31), the capacity of soluble antigen to absorb antibody to the same solid-phase antigen (38), different antibody binding patterns with different patient groups $(38,54)$, the uniform reactivity of monoclonal antibodies with different forms of the same antigen (39), and the same binding specificities of antibody Fab fragments (55).

binding was lower for this group than the previous one, although there were a few strong reactors. In these sera, reactivity to dimer-DNA and the three forms of chromatin predominated, whereas binding to H1-DNA was low and reactivity to tetramer-DNA* (and of course nDNA) virtually was absent. Four of 11 sera showed increased binding to the $\mathrm{H} 2 \mathrm{~A}-\mathrm{H} 2 \mathrm{~B}$ dimer compared with $\mathrm{H} 2 \mathrm{~A}$ or $\mathrm{H} 2 \mathrm{~B}$, although not as dramatic a change as the sera in Fig. $1 \mathrm{~A}$. Four displayed a substantial increase and five displayed a modest increase in binding to dimer-DNA compared with the $\mathrm{H} 2 \mathrm{~A}-\mathrm{H} 2 \mathrm{~B}$ dimer. The increase in binding between dimer and dimer-DNA can not be due to anti-nDNA antibodies because these sera did not display binding to nDNA (Fig. $1 B$ ). None of these sera showed an increase to (H3-
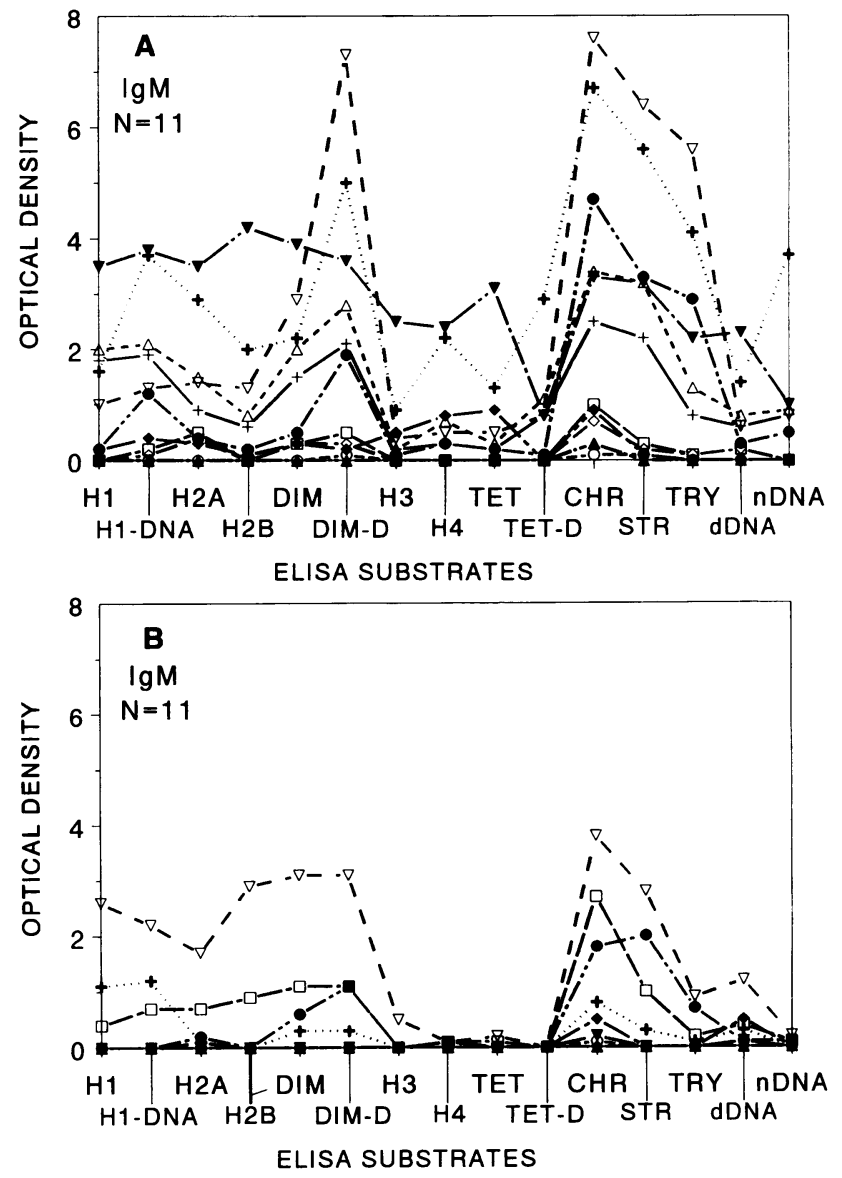

Figure 2. IgM autoantibodies to components of chromatin in SLE. The data points connected by a line are the activities (OD) of a serum on the indicated substrates after correcting for normal serum binding. $(A)$ IgM activities in the patients from Fig. $1 A$. $(B)$ IgM activities in the patients from Fig. $1 B$. Each patient is represented by the same symbol in Figs. 1 and $2 A$, and in Figs. 1 and $2 B$. The two figures are drawn to a different scale than Fig. 1. The abbreviations for the substrates are given in the legend to Fig. 1.

$\mathrm{H} 4)_{2}$ or $(\mathrm{H} 3-\mathrm{H} 4)_{2}$-DNA relative to the components of these complexes. All the sera in this group displayed the highest binding to whole chromatin. Many of these sera also bound to dDNA. Except for the four sera that bound $\mathrm{H} 1$, reactivity with individual histones was low or absent, particularly with $\mathrm{H} 3$ and $\mathrm{H} 4$.

IgM reactivities of the sera shown in Fig. $1 A$ are shown in Fig. $2 A$. Only a portion of their IgM was similar to their IgG reactivities. All 11 sera bound to whole chromatin and 10 bound to dimer-DNA. In contrast to their IgG, only five sera contained IgM that bound to nDNA or tetramer-DNA, reactivity to individual histones was relatively high compared with reactivity to chromatin, and one serum bound individual histones more strongly than histone-DNA complexes (Fig. $2 A$ ).

IgM binding in the group of sera without IgG anti-nDNA antibodies also showed predominant reactivity to chromatin (Fig. $2 B$ ). This group had less IgM antibodies than the group with IgG anti-nDNA, and four of the 11 sera were negative on all 15 substrates. There also was less IgM than IgG binding, since many sera displayed reactivity only to whole chromatin (compare Fig. $2 B$ with Fig. $1 B$ ). If IgG reactivity was lacking 
Table I. Amount and Prevalence of IgG and IgM Reactivity of SLE Sera on Chromatin and its Components

\begin{tabular}{|c|c|c|c|c|}
\hline \multirow[b]{2}{*}{ Substrate } & \multicolumn{2}{|c|}{ IgG } & \multicolumn{2}{|c|}{ IgM } \\
\hline & $\begin{array}{c}\text { Mean } \\
\text { OD } \pm \text { SD* }\end{array}$ & Prevalence ${ }^{\ddagger}$ & $\begin{array}{c}\text { Mean } \\
\mathrm{OD} \pm \mathrm{SD}^{*}\end{array}$ & Prevalence ${ }^{\ddagger}$ \\
\hline & & $\%$ & & $\%$ \\
\hline H1 & $1.2 \pm 1.9$ & 60 & $0.4 \pm 0.8$ & 35 \\
\hline H1-DNA & $2.6 \pm 4.3$ & 68 & $0.5 \pm 1.0$ & 42 \\
\hline $\mathrm{H} 2 \mathrm{~A}$ & $0.7 \pm 1.2$ & 55 & $0.4 \pm 0.8$ & 52 \\
\hline $\mathrm{H} 2 \mathrm{~B}$ & $1.0 \pm 1.6$ & 55 & $0.4 \pm 0.9$ & 30 \\
\hline $\mathrm{H} 2 \mathrm{~A}-\mathrm{H} 2 \mathrm{~B}$ dimer & $1.5 \pm 2.4$ & 68 & $0.6 \pm 1.0$ & 48 \\
\hline$(\mathrm{H} 2 \mathrm{~A}-\mathrm{H} 2 \mathrm{~B})-\mathrm{DNA}$ & $4.1 \pm 6.0$ & 70 & $0.8 \pm 1.6$ & 48 \\
\hline $\mathrm{H} 3$ & $0.3 \pm 0.6$ & 35 & $0.1 \pm 0.4$ & 22 \\
\hline H4 & $0.4 \pm 0.7$ & 38 & $0.2 \pm 0.5$ & 40 \\
\hline$(\mathrm{H} 3-\mathrm{H} 4)_{2}$-tetramer & $0.3 \pm 0.5$ & 45 & $0.2 \pm 0.5$ & 35 \\
\hline$(\mathrm{H} 3-\mathrm{H} 4)_{2}-\mathrm{DNA}$ & $1.4 \pm 3.0$ & 35 & $0.2 \pm 0.5$ & 20 \\
\hline Whole chromatin & $5.8 \pm 6.8$ & 88 & $1.2 \pm 1.8$ & 68 \\
\hline H1-stripped chromatin & $4.9 \pm 6.9$ & 78 & $0.8 \pm 1.5$ & 50 \\
\hline Trypsinized chromatin & $3.3 \pm 5.6$ & 60 & $0.5 \pm 1.2$ & 35 \\
\hline dDNA & $1.7 \pm 1.6$ & 82 & $0.4 \pm 0.5$ & 65 \\
\hline nDNA & $2.5 \pm 4.9$ & 55 & $0.2 \pm 0.6$ & 35 \\
\hline
\end{tabular}

* The average OD of 40 SLE sera after correcting for normal serum binding as described in Methods. ${ }^{\ddagger}$ The percentage of patients with reactivity $>$ mean +2 SD of 15 normal subjects.

in a serum, so was the corresponding $\operatorname{IgM}$ reactivity, except for anti-dDNA.

Average IgG and IgM binding to chromatin and its components. As seen in Table I, the average OD of IgG reactivity was highest for whole chromatin (5.8 OD), followed by H1-stripped chromatin, the (H2A-H2B)-DNA complex, and trypsinized chromatin (4.9-3.3 OD). The next highest level of reactivity was seen in the other DNA-containing substrates such as the subnucleosome structures $\mathrm{H} 1-\mathrm{DNA}$ and $(\mathrm{H} 3-\mathrm{H} 4)_{2}-\mathrm{DNA}$, as well as nDNA and dDNA (2.6-1.4 OD). Interestingly, the H2A-H2B dimer (1.5 OD) had comparable average OD and displayed the highest reactivity of the DNA-free substrates. The $(\mathrm{H} 3-\mathrm{H} 4)_{2}$ tetramer and the denatured individual histones $\mathrm{H} 3$ and $\mathrm{H} 4(0.3-0.4 \mathrm{OD})$ showed the lowest reactivity. The ranking of the substrates by percent of patients showing $>2$ SD above normal was generally the same as the ranking of average OD; that is, chromatin ( $88 \%$ positive), H1-stripped chromatin $(78 \%)$, and (H2A-H2B) -DNA (70\%) displayed the highest percent positive, and individual histones displayed the lowest. The only exception was anti-dDNA, in which $82 \%$ of the patients were positive, although their average reactivity was relatively modest.

For IgM autoantibodies, binding to chromatin (68\% positive ) still dominated, but the percentage of patients with reactivity to the DNA-free histones (22-52\%) was equal to or higher than the percent binding to the subnucleosome structures and nDNA (35-48\%) (Table I). The percentage of patients positive for an IgM reactivity was less than that for the corresponding IgG reactivity, except for $\mathrm{H} 4$. The ranking of average IgM OD on these substrates was very similar to their ranking by average IgG OD. That is, whole chromatin (1.2 OD), H1-stripped chromatin (0.8 OD), and (H2A-H2B ) -DNA (0.8 OD) were most reactive, whereas $\mathrm{H} 4(0.2 \mathrm{OD})$ and $\mathrm{H} 3(0.1 \mathrm{OD})$ were least reactive. There were two differences between IgG and IgM reactivities. The IgM binding to nDNA was relatively low $(0.2$ OD), and IgM binding to the individual histones tended to be one half the level displayed by the corresponding IgG antibodies, whereas IgM reactivity to native structures was only one fifth or less that of the IgG.

Statistical relationships between antibody reactivities. By visual examination of the data, IgG binding to nDNA and ( $\mathrm{H} 3-$ $\mathrm{H} 4)_{2}$-DNA seem related, as does IgG binding to dimer-DNA and chromatin (Figs. $1 A$ and $B$ ). We therefore compared antibody activities by linear regression analysis in all combinations of IgG substrates, IgM substrates, and IgG versus IgM. As expected, IgG binding to dimer-DNA was highly correlated to IgG binding to the three forms of chromatin $(r=0.94-0.97)$; less to dimer, tetramer-DNA, and nDNA $(r=0.78-0.83)$; and less again with H2B and H2A ( $r=0.64$ and 0.69 ). IgG nDNA showed very strong correlations with both IgG tetramerDNA $(r=0.96)$ and IgG H1-DNA $(r=0.91)$. When antinDNA was compared with reactivity with the three forms of chromatin, there was progressively less correlation with the more native forms ( $r=0.92$ with trypsinized chromatin, $r$ $=0.88$ with $\mathrm{H} 1$-stripped chromatin, and $r=0.76$ with whole chromatin).

For the four core histones (i.e., H2A, H2B, H3, and H4), the $r$ value was $>0.7$ when reactivity to any of the core histones was compared with any other core histone. In contrast, $\mathrm{H} 1$ stood out from the core histones by displaying a correlation coefficient $<0.7$ when tested with all other antigens except the $(\mathrm{H} 3-\mathrm{H} 4)_{2}$ tetramer. For the intra-IgG comparisons, no other correlations were $>0.7$. The intra-IgM comparisons were essentially the same as the intra-IgG comparisons described above. When interIgG/IgM comparisons were made, only anti-H1 $(r=0.85)$ and anti- $(\mathrm{H} 3-\mathrm{H} 4)_{2}$-DNA $(r=0.80)$ showed correlations $>0.7$. These statistical analyses confirm the significance (or lack of significance) of the relationships among this set of antigens that were apparent by visual examination of the data.

The correlation between anti-(dimer-DNA) and antichromatin was not a reflection of higher overall autoantibody levels in sera with elevated anti-(dimer-DNA) because these specificities showed no or only weak correlations with antibodies to individual histones. Additionally, we tested all sera for IgG antibodies to tetanus toxoid. The SLE sera had a mean \pm SD antitetanus activity of $1.47 \pm 1.86 \mathrm{OD}$ (range 0.01-5.96 OD). Correlation coefficients between antitetanus and anti-(dimerDNA) or antichromatin were 0.20 and 0.17 , respectively. Thus, the highly correlated autoantibody specificities were not due to a generally elevated immune status or hypergammaglobulinemia in patients with antichromatin antibodies.

Correlations between autoantibodies and symptoms. Almost all comparisons between a patient's IgG autoantibodies and disease symptoms were negative using chi-square analysis with Yate's correction for all 15 antigens and the 10 symptoms listed in Methods. The highest correlation was found between anti$(\mathrm{H} 2 \mathrm{~A}-\mathrm{H} 2 \mathrm{~B})$ and proteinuria $\left(\chi^{2}=10.6, P<0.01\right)$. AntiH1-stripped chromatin, anti-whole chromatin and anti-[(H2AH2B ) - DNA ] were also significantly correlated with proteinuria $\left(\chi^{2}=8.0,6.3\right.$, and 5.2 respectively, with $\left.P<0.05\right)$. The correlation of proteinuria with anti-nDNA was elevated, but not significantly $\left(\chi^{2}=2.4, P>0.1\right)$. Because a number of related antigens all displayed a strong correlation with proteinuria, and because anti-nDNA has been previously noted in SLE patients with renal disease, these statistical results seem reliable. How- 
ever, because a large number of comparisons were made, the few other correlations reaching $P<0.05$ may have occurred by chance alone and therefore may not be found for other patient populations. These were anti-H2B and alopecia, anti-H1stripped chromatin with neurologic symptoms, and anti-(H1DNA), $\mathrm{H} 2 \mathrm{~A}-\mathrm{H} 2 \mathrm{~B}$, and nDNA with oral ulcers.

Contribution of nDNA antibodies to the reactivity of histone-DNA complexes. Strong IgG binding to $(\mathrm{H} 2 \mathrm{~A}-\mathrm{H} 2 \mathrm{~B})-$ DNA and/or chromatin with no or low reactivity to nDNA and (H3-H4) $)_{2}$-DNA was observed in more than one third of the sera (Fig. $1 B$ ). However, sera containing IgG binding to nDNA reacted to $(\mathrm{H} 3-\mathrm{H} 4)_{2}-\mathrm{DNA}$ and $\mathrm{H} 1-\mathrm{DNA}$, as well as (H2AH2B)-DNA and chromatin (Fig. $1 A$ ). These relationships were confirmed by linear regression analysis (see above). To determine if reactivities to $(\mathrm{H} 3-\mathrm{H} 4)_{2}-\mathrm{DNA}$ and $\mathrm{H} 1-\mathrm{DNA}$ complexes solely were due to anti-nDNA antibodies binding to the DNA component of these antigens or if there were also antibodies specific for the protein-DNA complex, the 10 sera with the highest nDNA antibodies shown in Fig. $1 A$ were adsorbed with nDNA and tested on all DNA-containing substrates. After adsorption with nDNA, an average of only $12 \%$ of nDNA reactivity remained in the sera (Fig. $3 \mathrm{~A}$ ). However, an average of $46 \%$ of anti-(H1-DNA) and $57 \%$ of anti-(dimer-DNA) still remained. Only $36 \%$ of anti-(tetramer-DNA) was left, but $78 \%$ of the anti-whole chromatin activity still remained. Thus anti-nDNA antibodies did not account for most of the antichromatin and anti-(dimer-DNA) activities, as well as a substantial portion of the antibody activity against the ( $\mathrm{H} 3-\mathrm{H} 4)_{2}-\mathrm{DNA}$ and H1-DNA subnucleosome structures. In contrast, after adsorption with chromatin, only $4 \%$ to $13 \%$ of original activity remained for all substrates except H1-DNA, which still had an average of $38 \%$ activity remaining (Fig. 3 $B$ ). Similar results were obtained for all substrates when the adsorption was repeated. Thus, unlike nDNA, whole chromatin contains virtually all the antigenic activity recognized by antibodies to all chromatin substructures except H1-DNA.

Autoantibodies to chromatin and histones in American SLE patients. To determine whether the high prevalence of chromatin-reactive antibodies in this predominantly Chinese sample generalized to SLE patients of other racial demographics, we tested 37 American SLE patients with well-established disease for reactivity to various antigens. After correcting for normal serum binding, the percentage of patients with reactivity to the individual histones $\mathrm{H} 1$ and $\mathrm{H} 2 \mathrm{~B}$ was $24 \%$ and $41 \%$, respectively. Anti-dimer-DNA and anti-whole chromatin were detected in $59 \%$ and $70 \%$ of the patients, respectively. Only one serum had anti-DNA and even the average antichromatin activity was relatively moderate $(0.79 \pm 1.15 \mathrm{OD})$, probably because of the therapeutic intervention most of these patients received. Nevertheless, the predominance of anti-dimer-DNA/chromatin antibodies in this sample of American patients was similar to the serologic features of the patients obtained from Singapore.

Comparison of murine and human autoantibodies. In the MRL/lpr and BXSB strains of autoimmune mice, the pattern of chromatin-binding antibodies appears to be similar to that found in the SLE patients reported here. Specifically, antichromatin and anti-[(H2A-H2B $)-D N A]$ reactivity predominated, with or without concomitant anti-nDNA activity; binding to individual histones was relatively low; and reactivity with nDNA and (H3-H4) $)_{2}$-DNA were linked (39). In addition, patients with procainamide-induced lupus have antibodies that are primarily directed against the $\mathrm{H} 2 \mathrm{~A}-\mathrm{H} 2 \mathrm{~B}$ dimer (42), the
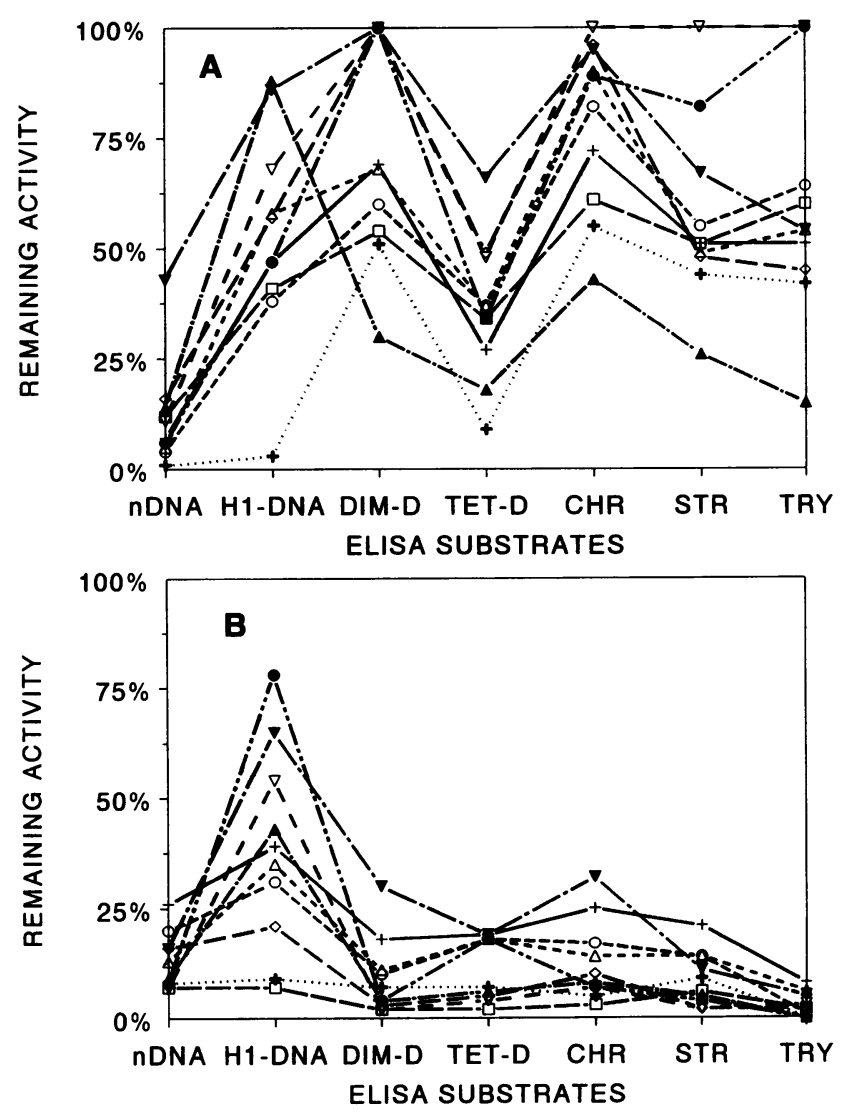

Figure 3. Solid-phase adsorption with chromatin or nDNA. Ten of the sera from Fig. $1 A$ were adsorbed with $\mathrm{nDNA}(A)$ or whole chromatin $(B)$ and the remaining IgG binding on seven DNA-containing substrates was determined. The patients are represented by the same symbols that identified them in Fig. $1 \mathrm{~A}$. The abbreviations for the substrates are given in the legend to Fig. 1.

(H2A-H2B ) -DNA subnucleosome, and chromatin, with no or much lower reactivity against other subnucleosome structures, nDNA, or individual histones (38). To compare the antibody specificities in these groups, serum from a typical SLE patient with anti-nDNA specificity and from a procainamide-induced lupus patient were tested for their ability to block murine antichromatin autoantibodies. The procainamide-induced lupus serum could block murine sera that did not have anti-nDNA an average of $94 \%$, but sera with anti-nDNA activity only $62 \%$ (Table II). The SLE serum with anti-nDNA blocked $98 \%$ and $82 \%$ of the reactivity from the two sets of mouse sera, respectively. The inability of the procainamide-induced lupus serum to block the binding of monoclonal antibodies to nDNA or histone H2B and the only partial blockage of the anti-nDNA monoclonal antibodies by the SLE serum demonstrated that blocking of mouse sera was not simply due to stearic hindrance.

\section{Discussion}

In the current study, $75 \%$ of the Singapore SLE patients had IgG antibodies to at least one histone. Much of this reactivity was very low, and only $55 \%$ were positive at $>0.1$ OD above normal, in good agreement with the average prevalence of $51 \%$ in previous studies (43). However, antibody binding to more 
Table II. Inhibition of Murine Antichromatin Autoantibodies by Human Sera

\begin{tabular}{lccc}
\hline & \multicolumn{3}{c}{ Anti-H1-stripped chromatin reactivity } \\
\cline { 2 - 4 } & \multicolumn{4}{c}{$\begin{array}{c}\text { Blocking by human } \\
\text { serum }\end{array}$} \\
\cline { 2 - 4 } & & \multicolumn{3}{c}{$\%$} \\
\cline { 2 - 4 } Origin of antibody & Control OD* & $\begin{array}{c}\text { Procainamide- } \\
\text { induced LE }\end{array}$ & SLE \\
No. 23 \\
\hline
\end{tabular}

* Antibody reactivity of sera from various autoimmune mice (39) or monoclonal antibodies $(40,41)$ on $\mathrm{H1}$-stripped chromatin in the presence of normal human serum. ${ }^{\ddagger}$ The procainamide-induced lupus serum contained antichromatin but not anti-nDNA antibodies. SLE No. 23 serum contained antibodies of both specificities.

native components of chromatin was enhanced, reaching $88 \%$ positive for whole chromatin as the test antigen. Moreover, the level of IgG reactivity for whole chromatin was more than eight times the average reactivity of individual histones. Similar results were obtained in a limited study of American patients with well-established SLE receiving antiinflammatory treatment, although the levels of autoantibodies in this group of patients was lower. Thus, there was no indication of a racial or ethnic association of antichromatin antibodies as has been reported for antibodies to Sm and nRNP (44). It appears, therefore, that antichromatin antibodies are the most common autoantibody specificity in SLE, and, based on direct binding, correlation analyses, and adsorption studies, the (H2A-H2B)-DNA subnucleosome component appears to account for the bulk of this activity.

Some of the increased antigenicity of chromatin could have been due to its more complex macromolecular composition, since it consists of five different histones and nDNA. However, in sera with anti-nDNA, adsorption studies demonstrated that $<25 \%$ of antichromatin reactivity was due to anti-nDNA antibodies. Additionally, about one third of the sera had high antichromatin reactivity with relatively little anti-nDNA or antihistone. Therefore, the bulk of antichromatin antibodies in most SLE sera react with epitopes created by the tertiary and quater- nary structures formed by histone-histone and histone-DNA interactions, and antibodies to individual histones or to nDNA were minor components of the IgG antichromatin response.

Often a serum demonstrated IgG but not IgM binding to some of the substrates tested, but very rarely was there IgM reactivity in the absence of IgG. Most IgM and IgG antibodies were not significantly correlated, consistent with previous reports for individual histones $(5,45)$. The predominant IgG and IgM reactivity was directed against whole chromatin. However, there was strong IgG binding to all the native components of chromatin, whereas IgM did not display this pattern so prominently. These results could be explained by the existence in vivo of a highly potent antigen drive that results in IgM to IgG class switch accompanied by altered V-region specificity during affinity maturation.

In some studies, the presence of anti-nDNA has been correlated with the severity of disease and the presence of lupus nephritis $(25,46,47)$, especially if changes in antibody levels over time are considered $(48,49)$. In other studies no significant correlation between disease and anti-nDNA was found $(50,51)$. Usually reactivity to individual histones has not been correlated with disease $(45,52)$. We found that anti- $(\mathrm{H} 2 \mathrm{~A}-\mathrm{H} 2 \mathrm{~B})$, anti[(H2A-H2B $)-D N A]$ and antichromatin were significantly correlated with proteinuria whereas other reactivities were not. These results suggest that anti-(H2A-H2B) and antichromatin antibodies could contribute to glomerulonephritis and thus might have some diagnostic utility in SLE. However, it should be mentioned that the correlation was not perfect, since 5 of the 10 patients with the highest antichromatin reactivity did not display proteinuria. Protein in the urine is not a sensitive indication of all forms of renal disease because glomerular damage as judged by microscopic examination of biopsies was not well correlated to proteinuria (47). Renal biopsies were not performed in the present study. It was interesting that anti( $\mathrm{H} 2 \mathrm{~A}-\mathrm{H} 2 \mathrm{~B})$ yielded the highest correlation with proteinuria, since it has been suggested that histone binding to glomeruli may be important in developing antibody-induced glomerulonephritis (53).

Some sera had antibodies reactive with chromatin and (H2A-H2B ) -DNA but not with nDNA or (H3-H4) $)_{2}-\mathrm{DNA}$. Other sera displayed binding to chromatin and (H2A-H2B)DNA as well as nDNA and ( $\mathrm{H} 3-\mathrm{H} 4)_{2}-\mathrm{DNA}$. These latter sera were adsorbed with nDNA to determine if their binding to nDNA accounted for their reactivity to the other DNA-containing substrates. About three quarters of the original binding to whole chromatin and half of the original binding to dimerDNA, tetramer-DNA, and H1-DNA remained after nDNA reactivity was removed. Antibodies in these sera thus recognized many epitopes on chromatin, including those present on nDNA and all three subnucleosome particles.

These sera were also adsorbed with chromatin to determine if chromatin could remove the reactivity to the subnucleosome particles or if these substrates possessed antigenic regions not present on chromatin. Specifically, there are regions of the (H2A-H2B ) -DNA complex and the (H3-H4) $)_{2}$-DNA complex that are buried in chromatin and not exposed to solution $(27,28)$. As previously demonstrated, we detected IgM antibodies induced by chlorpromazine and hydralazine that recognized epitopes on these regions (38). On the chromatin-related substrates, these drug-induced lupus sera reacted with histoneDNA subnucleosomes but not with chromatin, and adsorption with chromatin did not remove these antibodies (38). In con- 


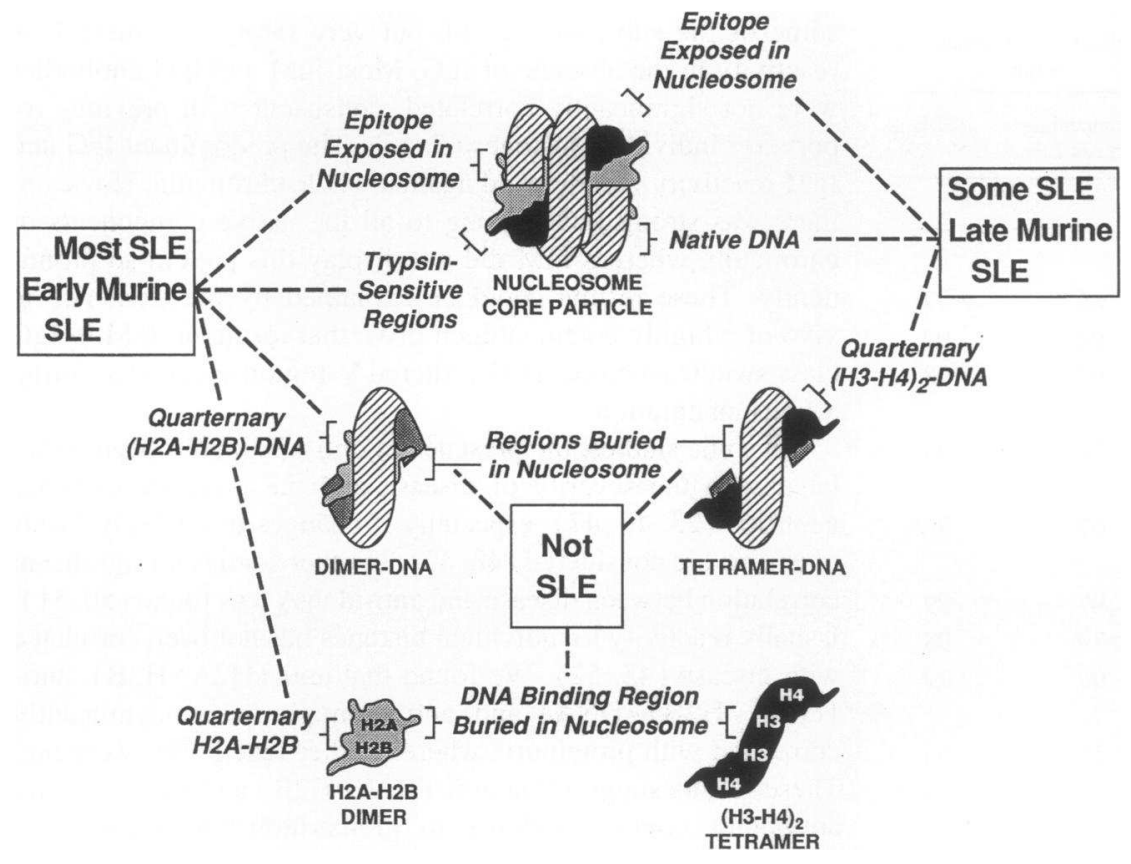

Figure 4. Relationship between autoantibody specificity and nucleosome structure. The nucleosome core particle, the repeating unit in $\mathrm{H1}$ stripped chromatin used as an antigen in this study, is based on the structure determined by $x$ ray crystallography $(27,28)$. On the left side of the picture the epitopes common to human and murine lupus with antichromatin antibodies are bracketed: i.e., from top to bottom, regions of the H2A-H2B dimer exposed in chromatin; the histone tails sensitive to digestion with trypsin; regions in (H2A-H2B)-DNA requiring quaternary protein-DNA interaction; and regions of the dimer requiring quaternary protein-protein interactions. On the right side of the picture, the epitopes recognized by the subset of human and murine sera that also bind to nDNA are bracketed: i.e., the part of the (H3-H4) $)_{2}$-DNA complex exposed in chromatin, and nDNA itself. In the middle of the picture the regions of the nucleosome that are not antigenic in lupus sera are bracketed: i.e., the regions of $(\mathrm{H} 2 \mathrm{~A}-\mathrm{H} 2 \mathrm{~B})-$ DNA and $(\mathrm{H} 3-\mathrm{H} 4)_{2}-$ DNA that are buried in chromatin by protein-protein interactions and the regions of $\mathrm{H} 2 \mathrm{~A}-\mathrm{H} 2 \mathrm{~B}$ and $(\mathrm{H} 3-\mathrm{H} 4)_{2}$ covered by DNA in chromatin. Other antibodies to individual histones are not indicated because such activity is relatively low and the location of those epitopes on the nucleosome is unclear. trast, absorption of the SLE sera with chromatin removed almost all binding to the $(\mathrm{H} 2 \mathrm{~A}-\mathrm{H} 2 \mathrm{~B})-\mathrm{DNA}$ and $(\mathrm{H} 3-\mathrm{H} 4)_{2}-\mathrm{DNA}$ subnucleosome components, demonstrating that the regions of subnucleosomes buried in chromatin are not antigenic in SLE. The relationship between the antigenic specificities described above and nucleosome structure is depicted in Fig. 4.

Reactivities to H1-stripped chromatin, trypsinized chromatin, and nDNA also were removed by adsorption with whole chromatin. These results are most consistent with the concept that whole chromatin, but not isolated subnucleosome structures, is an immunogenic stimulus for B cells in SLE, and nDNA antibodies are a subset of the wide range of antichromatin autoantibodies produced in this disease.

The full range of chromatin components has not been previously tested with sera from patients with SLE, and this study revealed two related patterns of IgG reactivity in virtually all the patients. For both patterns, native substrates such as whole chromatin, H1-stripped chromatin, and the (H2A-H2B ) -DNA complex were most reactive, whereas the denatured individual histones, particularly $\mathrm{H} 3$ and $\mathrm{H} 4$, were least reactive. Poor antigenicity of $\mathrm{H} 3$ and $\mathrm{H} 4$ has been reported previously (for review see reference 43 ). The patterns were not caused by assay bias since similar amounts of all substrates are bound to the ELISA plate (31), and non-SLE sera yielded different patterns on the identical substrates $(38,54)$. In agreement with studies using the Western blot technique $(4,6,7)$, the trypsin-sensitive regions of histones were often antigenic, since trypsinized chromatin usually displayed lower reactivity than H1-stripped chromatin. However, most sera displayed substantially stronger reactivity to trypsinized chromatin than to individual histones, indicating that the major epitopes in SLE sera occur in the trypsin-resistant regions of chromatin.

The patterns of antibody reactivity to components of chro- matin in patients with SLE were remarkably similar to those of the MRL/lpr and BXSB strains of lupus-prone mice (39). These mice developed antichromatin and anti-[(H2A-H2B $)-$ DNA] antibodies early in life, and $\sim 75 \%$ of them went on to develop anti-nDNA and anti-[(H3-H4) $)_{2}-$ DNA $]$ later in the course of their disease. SLE patients with only anti-[(H2AH2B ) -DNA] and antichromatin resembled the pattern from young mice at the initial appearance of autoantibodies. Those patients with antibodies to nDNA and the (H3-H4) $)_{2}-\mathrm{DNA}$ subnucleosome had a serology like that of the older mice.

The similarity of the murine and human autoantibodies was supported by inhibition studies in which serum from an SLE patient with anti-nDNA inhibited virtually all of the binding to chromatin of both young and old mice. Serum from a procainamide-induced lupus patient that contained only antichromatin blocked the murine antichromatin but not the murine anti-DNA antibodies. Thus, human and murine autoantibodies recognized overlapping or identical epitopes on chromatin, implying that the fundamental immunologic stimulus causing antichromatin autoantibodies is the same in both species.

Patients with lupus induced by procainamide (38) and certain other drugs (55) also showed predominant reactivity to chromatin and (H2A-H2B)-DNA but not nDNA or (H3$\mathrm{H} 4)_{2}$-DNA. An $\mathrm{F}\left(\mathrm{ab}^{\prime}\right)_{2}$ fragment from a patient with procainamide-induced lupus blocked $>90 \%$ of the anti-[(H2AH2B ) -DNA] reactivity in six of six sera from other patients with procainamide-induced lupus, four of four with quinidineinduced lupus, as well as sera from patients with lupus induced by acebutolol, penicillamine, and isoniazid (55). Thus, autoantibodies from humans with SLE, two strains of lupus-prone mice, and patients with lupus caused by five different drugs all recognize identical or overlapping epitopes on chromatin.

Our interpretation of this finding is that these individuals 
may have been exposed to a similar immunologic event. Autoimmunization with chromatin could account for the antibody profiles described in this study, but the mechanism causing chromatin to become immunogenic is obscure. However, once tolerance to an epitope on chromatin is broken, B cells may process chromatin and present histone peptides to $\mathrm{T}$ cells, which may then provide help for secretion of anti-nDNA and other antichromatin autoantibodies (56). The relatively low reactivity to individual histones compared with higher order structures is consistent with chromatin being the immunogen. The pattern of high reactivity to chromatin and $(\mathrm{H} 2 \mathrm{~A}-\mathrm{H} 2 \mathrm{~B})-\mathrm{DNA}$ but not nDNA or ( $\mathrm{H} 3-\mathrm{H} 4)_{2}$-DNA could arise if the (H2A-H2B)DNA region in chromatin became immunogenic in an environment where B cell tolerance to the ( $\mathrm{H} 3-\mathrm{H} 4)_{2}-$ DNA subnucleosome and nDNA was preserved. This is exactly the pattern seen in young lupus-prone mice, in patients with drug-induced lupus, and in about one third of the SLE patients. Appearance of antibodies to nDNA and the (H3-H4) $)_{2}$-DNA and H1-DNA subnucleosomes could be explained by subsequent spreading of the immune response to all portions of chromatin. Such a profile of reactivity characterizes $75 \%$ of older lupus-prone mice and $\sim 50 \%$ of the SLE patients. We suggest that SLE patients with anti-nDNA represent later stage disease when global loss of tolerance to numerous epitopes on chromatin occurs.

\section{Acknowledgments}

We thank Drs. D. Kono and Dr. M. Duchosal for critically reading the manuscript and Dr. A. N. Theofilopoulos for sera from autoimmune mice. Dr. Burlingame was a fellow of the Arthritis Foundation. This is publication number 8164-MEM from The Scripps Research Institute, La Jolla.

This work was supported by grant ES06334 from the National Institute of Environmental Health Sciences, National Institutes of Health, National Institutes of Health grant MO1-RR00833 and a grant from the Medical Research Service of the Department of Veterans Affairs (G. Starkebaum).

\section{References}

1. Autoantibodies. Section IV. 1993. In Dubois' Lupus Erythematosus. D. J. Wallace, B. H. Hahn, F. P. Quismorio, and J. R. Klinenberg, editors. Lea and Febiger, Philadelphia. 181-276.

2. Tan, E. M. 1989. Antinuclear antibodies: diagnostic markers for autoimmune diseases and probes for cell biology. Adv. Immunol. 44:93-151.

3. Fritzler, M. J., and E. M. Tan. 1978. Antibodies to histones in drug-induced and idiopathic lupus erythematosus. J. Clin. Invest. 62:560-567.

4. Thomas, J. O., C. M. Wilson, and J. A. Hardin. 1984. The major core histone antigenic determinants in systemic lupus erythematosus are in the trypsinsensitive regions. FEBS (Fed. Eur. Biochem. Soc.) Lett. 169:90-96.

5. Krippner, H., B. Springer, S. Merle, and K. Pirlet. 1984. Antibodies to histones of the IgG and IgM class in systemic lupus erythematosus. Clin. Exp. Immunol. 58:49-56.

6. Gohill, J., P. D. Cary, M. Couppez, and M. J. Fritzler. 1985. Antibodies from patients with drug-induced and idiopathic lupus erythematosus react with epitopes restricted to the amino and carboxyl termini of histone. J. Immunol. 135:3116-3121.

7. Portanova, J. P., R. E. Arndt, E. M. Tan, and B. L. Kotzin. 1987. Antihistone antibodies in idiopathic and drug-induced lupus recognize distinct intrahistone regions. J. Immunol. 138:446-451.

8. Tan, E. M., and P. G. Natali. 1970. Comparative study of antibodies to native and denatured DNA. J. Immunol. 104:902-906.

9. Arana, R., and M. Seligmann. 1967. Antibodies to native and denatured deoxyribonucleic acid in systemic lupus erythematosus. J. Clin. Invest. 46:18671882.

10. Schwartz, R. S., and B. D. Stollar. 1985. Origins of anti-DNA autoantibodies. J. Clin. Invest. 75:321-327.

11. Northway, J. D., and E. M. Tan. 1972. Differentiation of antinuclear antibodies giving speckled staining patterns in immunofluorescence. Clin. Immunol. Immunopathol. 1:140-146.

12. Habets, W. J., M. H. Hoet, and W. J. van Venrooij. 1990. Epitope patterns of anti-RNP antibodies in theumatic diseases. Evidence for an antigen-driven autoimmune response. Arthritis Rheum. 33:834-841.

13. Reichlin, M., and M. W. Reichlin. 1989. Autoantibodies to the Ro/SS-A particle react preferentially with the human antigen. J. Autoimmun. 2:359-365.

14. Shlomchik, M., M. Mascelli, H. Shan, M. Z. Radic, D. Pisetsky, A. Marshak-Rothstein, and M. Weigert. 1990. Anti-DNA antibodies from autoimmune mice arise by clonal expansion and somatic mutation. J. Exp. Med. 171:265-297.

15. Marion, T. N., D. M. Tillman, and N.-T. Jou. 1990. Interclonal and intraclonal diversity among anti-DNA antibodies from an (NZBXNZW) $\mathrm{F}_{1}$ mouse. J. Immunol. 145:2322-2332.

16. Benjamin, D. C., J. A. Berzofsky, I. J. East, F. R. N. Gurd, C. Hannum, S. J. Leach, E. Margoliash, J. G. Michael, A. Miller, E. M. Prager, et al. 1984. The antigenic structure of proteins: a reappraisal. Annu. Rev. Immunol. 2:67-101.

17. Rubin, R. L., F.-L. Tang, G. Tsay, and K. M. Pollard. 1990. Pseudoautoimmunity in normal mice: anti-histone antibodies elicited by immunization versus induction during graft-versus-host reaction. Clin. Immunol. Immunopathol. 54:320-332.

18. Madaio, M. P., S. Hodder, R. S. Schwartz, and B. D. Stollar. 1984. Responsiveness of autoimmune and normal mice to nucleic acid antigens. $J$. Immunol. 132:872-876.

19. Oldstone, M. B. A. 1987. Molecular mimicry and autoimmune disease. Cell. 50:819-820.

20. Pisetsky, D. S., J. P. Grudier, and G. S. Gilkeson. 1990. A role for immunogenic DNA in the pathogenesis of systemic lupus erythematosus. Arthritis Rheum. 33:153-159.

21. Klinman, D. M., and A. D. Steinberg. 1987. Systemic autoimmune disease arises from polyclonal B cell activation. J. Exp. Med. 165:1755-1760.

22. Harkiss, G. D., and B. H. Hahn. 1990. The characterization of DNA antibody idiotypes-a discussion. J. Autoimmun. 3:367-375.

23. Holman, H., and H. R. Deicher. 1959. The reaction of the lupus erythematosus (LE) cell factor with deoxyribonucleoprotein of the cell nucleus. J. Clin. Invest. 38:2059-2072.

24. Rekvig, O. P., and K. Hannestad. 1981. Lupus erythematosus (LE) factors recognize both nucleosomes and viable human leukocytes. Scand. J. Immunol. 13:597-604.

25. Robitaille, P., and E. M. Tan. 1973. Relationship between deoxyribonucleoprotein and deoxyribonucleic acid antibodies in systemic lupus erythematosus. J. Clin. Invest. 52:316-323.

26. Stollar, B. D. 1971. Reactions of systemic lupus erythematosus sera with histone fractions and histone-DNA complexes. Arthritis Rheum. 4:485-492.

27. Richmond, T. J., J. T. Finch, B. Rushton, D. Rhodes, and A. Klug. 1984. Structure of the nucleosome core particle at 7 A resolution. Nature (Lond.) 311:532-537.

28. Arents, G., R. W. Burlingame, B.-C. Wang, W. E. Love, and E. N. Moudrianakis. 1991. The nucleosomal core histone octamer at $3.1 \mathrm{~A}$ resolution: a tripartite protein assembly and a left-handed superhelix. Proc. Natl. Acad. Sci. USA. 88:10148-10152.

29. Godfrey, J. E., A. D. Baxevanis, and E. N. Moudrianakis. 1990. Spectropolarimetric analysis of the core histone octamer and its subunits. Biochemistry. 29:965-972.

30. Mencke, A. J., and R. L. Rill. 1982. Circular dichroism and thermal denaturation studies of subnucleosomes and their relationships to nucleosome structure. Biochemistry. 21:4362-4370.

31. Burlingame, R. W., and R. L. Rubin. 1990. Subnucleosome structures as substrates in enzyme-linked immunosorbent assays. J. Immunol. Methods. 134:187-199.

32. Lutter, L. C. 1978. Kinetic analysis of deoxyribonuclease I cleavages in the nucleosome core: evidence for a DNA superhelix. J. Mol. Biol. 124:391420 .

33. Weintraub, H., and F. Van Lente. 1974. Dissection of chromosome structure with trypsin and nucleases. Proc. Natl. Acad. Sci. USA. 71:4249-4253.

34. Tan, E. M., A. S. Cohen, J. F. Fries, A. T. Masi, D. J. McShane, N. F. Rothfield, J. G. Schaller, N. Talal, and R. J. Winchester. 1982. The 1982 revised criteria for the classification of systemic lupus erythematosus. Arthritis Rheum. 25:1271-1277.

35. Boey, M. L., C. L. Peebles, G. Tsay, P. H. Feng, and E. M. Tan. 1988. Clinical and autoantibody correlations in Orientals with systemic lupus erythematosus. Ann. Rheum. Dis. 47:918-923.

36. Oohara, I., and A. Wada. 1987. Spectroscopic studies on histone-DNA interactions I. The interaction of histone (H2A, H2B) dimer with DNA: DNA sequence dependence. J. Mol. Biol. 196:389-397.

37. van der Westhuyzen, D. R., and C. Von Holt. 1971. A new procedure for the isolation and fractionation of histones. FEBS (Fed. Eur. Biochem. Soc.) Lett. 14:333-337.

38. Burlingame, R. W., and R. L. Rubin. 1991. Drug-induced anti-histone 
autoantibodies display two patterns of reactivity with substructures of chromatin. J. Clin. Invest. 88:680-690.

39. Burlingame, R. W., R. L. Rubin, R. S. Balderas, and A. N. Theofilopoulos. 1993. The genesis and evolution of antichromatin autoantibodies in murine lupus implicates T-dependent immunization with self antigen. J. Clin. Invest. 91:16871696.

40. Kotzin, B. L., J. A. Lafferty, J. P. Portanova, R. L. Rubin, and E. M. Tan. 1984. Monoclonal anti-histone autoantibodies derived from murine models of lupus. J. Immunol. 133:2554-2559.

41. Rubin, R. L., and A. N. Theofilopoulos. 1987. Monoclonal autoantibodies reacting with multiple structurally related and unrelated macromolecules. Int. Rev. Immunol. 3:71-95.

42. Totoritis, M. C., E. M. Tan, E. M. McNally, and R. L. Rubin. 1988. Association of antibody to histone complex H2A-H2B with symptomatic procainamide-induced lupus. N. Engl. J. Med. 318:1431-1436.

43. Rubin, R. L. 1992. Anti-histone antibodies. In Systemic Lupus Erythematosus. R. G. Lahita, editor. John Wiley, New York. 247-271.

44. Arnett, F. C., R. G. Hamilton, M. G. Rober, J. B. Harley, and M. Reichlin 1988. Increased frequencies of Sm and nRNP autoantibodies in American black compared to whites with systemic lupus erythematosus. J. Rheumatol. 15:17731776.

45. Gompertz, N. R., D. A. Isenberg, and B. M. Turner. 1990. Correlation between clinical features of systemic lupus erythematosus and levels of antihistone antibodies of the IgG, IgA, and IgM isotypes. Ann. Rheum. Dis. 49:524-527.

46. Koffler, D., R. I. Carr, V. Agnello, R. Thoburn, and H. G. Kunkel. 1971. Antibodies to polynucleotides in human sera: antigenic specificity and relation to disease. J. Exp. Med. 134:294-312.

47. Okamura, M., Y. Kanayama, K. Amastu, N. Negoro, S. Kohda, T. Takeda, and T. Inoue. 1993. Significance of enzyme linked immunosorbent assay (ELISA) for antibodies to double stranded and single stranded DNA with patients with lupus nephritis: correlation with severity of renal histology. Ann. Rheum. Dis. 52:14-20.

48. Ludivico, C. L., B. Zweiman, A. R. Myers, J. Hebert, and P. A. Green. 1980. Predictive value of anti-DNA antibody and selected laboratory studies in systemic lupus erythematosus. J. Rheumatol. 7:843-849.

49. ter Borg, E. J., G. Horst, E. Hummel, P. C. Limburg, and C. G. M. Kallenberg. 1991. Rises in anti-double stranded DNA antibody levels prior to exacerbations of systemic lupus erythematosus are not merely due to polyclonal B cell activation. Clin. Immunol. Immunopathol. 59:117-128.

50. Gladman, D. D., M. B. Urowitz, and E. C. Keystone. 1979. Serologically active clinically quiescent systemic lupus erythematosus: a discordance between clinical and serological features. Am. J. Med. 66:210-215.

51. Isenberg, D. A., Y. Shoenfeld, and R. S. Schwartz. 1984. Multiple serologic reactions and their relationship to clinical activity in systemic lupus erythematosus. Arthritis Rheum. 27:132-138.

52. Cohen, M. G., K. M. Pollard, and J. Webb. 1992. Antibodies to histones in systemic lupus erythematosus: prevalence, specificity, and relationship to clinical and laboratory features. Ann. Rheum. Dis. 51:61-66.

53. Schmiedeke, T. M. J., F. W. Stockl, R. Weber, Y. Sugisaki, S. R. Bratsford, and A. Vogt. 1989. Histones have high affinity for the glomerular basement membrane: relevance for immune complex formation in lupus nephritis. J. Exp. Med. 169:1879-1894.

54. Burlingame, R. W., and R. L. Rubin. 1992. Anti-histone autoantibodies recognize centromeric heterochromatin in metaphase chromosomes and hidden epitopes in interphase cells. Hum. Antibod. Hybridomas. 3:40-47.

55. Rubin, R. L., S. A. Bell, and R. W. Burlingame. 1992. Autoantibodies associated with lupus induced by diverse drugs target a similar epitope in the (H2A-H2B)-DNA complex. J. Clin. Invest. 90:165-173.

56. Mohan, C., S. Adams, V. Stanik, and S. K. Datta. 1993. Nucleosome: a major immunogen for pathogenic autoantibody-inducing T cells of lupus. J. Exp. Med. 177:1367-1381. 